\title{
Tissue Preparation Techniques for Contrast-Enhanced Micro Computed Tomography Imaging of Large Mammalian Cardiac Models with Chronic Disease
}

\author{
Néstor Pallares-Lupon ${ }^{1,2}$, Jason D. Bayer ${ }^{1,2}$, Bastien Guillot ${ }^{1,2}$, Guido Caluori ${ }^{1,2}$, Girish S. Ramlugun ${ }^{1,2}$, Kanchan \\ Kulkarni $^{1,2}$, Virginie Loyer ${ }^{1,2}$, Stephane Bloquet ${ }^{1,2}$, Dounia El Hamrani ${ }^{1,2}$, Jérôme Naulin ${ }^{1,2}$, Marion Constantin ${ }^{1,2}$, Pierre \\ Dos Santos $^{1,2,3}$, Olivier Bernus ${ }^{1,2}$, Pierre Jaïs ${ }^{1,2,3}$, Philippe Pasdois ${ }^{1,2}$, Richard D. Walton ${ }^{1,2}$ \\ ${ }^{1}$ Centre de recherche Cardio-Thoracique de Bordeaux, Univ. Bordeaux ${ }^{2} \mathrm{IHU}$ Liryc, Electrophysiology and Heart Modeling Institute, Fondation Bordeaux \\ Univ. ${ }^{3}$ Electrophysiology and Ablation Unit, Bordeaux University Hospital (CHU)
}

\section{Corresponding Author}

Richard D. Walton

richard.walton@ihu-liryc.fr

\section{Citation}

Pallares-Lupon, N., Bayer, J.D.

Guillot, B., Caluori, G., Ramlugun, G.S., Kulkarni, K., Loyer, V., Bloquet, S., El Hamrani, D., Naulin, J., Constantin, M., Dos Santos, P., Bernus, O., Jaïs, P., Pasdois, P., Walton, R.D. Tissue Preparation Techniques for ContrastEnhanced Micro Computed Tomography Imaging of Large Mammalian Cardiac Models with Chronic Disease. J. Vis. Exp. (180), e62909, doi:10.3791/62909 (2022).

\section{Date Published}

February 8, 2022

DOI

$10.3791 / 62909$

URL

jove.com/video/62909

\section{Abstract}

Structural remodeling is a common consequence of chronic pathological stresses imposed on the heart. Understanding the architectural and compositional properties of diseased tissue is critical to determine their interactions with arrhythmic behavior. Microscale tissue remodeling, below the clinical resolution, is emerging as an important source of lethal arrhythmia, with high prevalence in young adults. Challenges remain in obtaining high imaging contrast at sufficient microscale resolution for preclinical models, such as large mammalian whole hearts. Moreover, tissue composition-selective contrast enhancement for three-dimensional highresolution imaging is still lacking. Non-destructive imaging using micro-computed tomography shows promise for high-resolution imaging. The objective was to alleviate sufferance from X-ray over attenuation in large biological samples. Hearts were extracted from healthy pigs $(N=2)$, and sheep $(N=2)$ with either induced chronic myocardial infarction and fibrotic scar formation or induced chronic atrial fibrillation. Excised hearts were perfused with: a saline solution supplemented with a calcium ion quenching agent and a vasodilator, ethanol in serial dehydration, and hexamethyldisilizane under vacuum. The latter reinforced the heart structure during air-drying for 1 week. Collagen-dominant tissue was selectively bound by an X-ray contrast-enhancing agent, phosphomolybdic acid. Tissue conformation was stable in air, permitting long-duration microcomputed tomography acquisitions to obtain highresolution (isotropic $20.7 \mu \mathrm{m}$ ) images. Optimal contrast agent loading by diffusion showed selective contrast enhancement of the epithelial layer and sub-endocardial Purkinje fibers in healthy pig ventricles. Atrial fibrillation (AF) hearts showed enhanced contrast accumulation in the posterior walls and appendages of the atria, attributed to greater collagen content. Myocardial infarction hearts showed increased 
contrast selectively in regions of cardiac fibrosis, which enabled the identification of interweaving surviving myocardial muscle fibers. Contrast-enhanced air-dried tissue preparations enabled microscale imaging of the intact large mammalian heart and selective contrast enhancement of underlying disease constituents.

\section{Introduction}

Structural heart disease accounts for the majority of cardiac-related mortality world-wide ${ }^{1}$. Remodeling of cardiac structure influences the myocardial environment and the interstitial space. Since both cardiac electrical and mechanical function depends on myocyte organization, disruption can lead to intolerable cardiac arrhythmia, impaired blood-pumping actions, and heart failure $2,3,4,5,6,7,8,9$. Developments of curative therapies for structural heart diseases are far outweighed by the disease prevalence ${ }^{2,5}$. As such, increasing numbers of preclinical models of structural cardiac diseases are emerging to better understand the anatomomorphological profiles and resulting pathogenesis of cardiac arrhythmias $10,11,12,13,14,15,16,17,18,19,20,21,22,23$.

Observed across the structural disease spectrum is the upregulation of interstitial fibrosis and, more commonly in ischemia-related cases, myocardial replacement by fibrosis and fat tissue ${ }^{18}$. Morphological understanding of pathological extracellular components can enable the identification of potential substrates of arrhythmia. The distribution and extent of the disease provide strong indicators of arrhythmogenic risk. Yet, challenges remain to comprehensively image disease profiles by integrating macro- and microscales in the intact heart.

Micro-computed tomography (microCT), based on X-rays, is emerging as a powerful tool to interrogate soft biological tissue microstructure using contrast agents. Highly detailed anatomical maps have been obtained for hearts from small rodents $24,25,26$ and small dissected samples from large mammalian hearts ${ }^{27,28}$. However, imaging at the whole organ level of large mammalian hearts presents excessive path lengths over which X-ray photons are attenuated using conventional tissue preparation techniques. This involves contrast-loading the tissue and immersing the sample in a contrast agent solvent during acquisition. Increasing the sample size and resolution imposes a prolongation of the total acquisition time. Therefore tissue stability becomes crucial for useable image reconstruction, meaning that tissue deformation resulting from drying must be prevented. The use of an immersion fluid, however, has drawbacks: (i) the overall background signal intensity becomes non-negligible and (ii) promotes dilution of tissue-bound contrast molecules. Both of these factors contribute to lowering image contrast.

This study details a novel tissue processing pipeline to alleviate background photon attenuation and optimize the dynamic range afforded by contrast-enhancement agents. It is suggested to use a tissue air-drying approach with chemical tissue reinforcement to limit tissue deformation ${ }^{29}$. Therefore tissue samples can remain stable in air for long acquisitions and omit background contributions from immersion fluids. This methodology pipeline provides: (i) a comprehensive tissue processing and imaging protocol optimized using whole pig hearts; (ii) an evaluation of contrast concentration and loading techniques and, (iii) application of this pipeline 
in two distinct chronic disease models of atrial fibrillation and myocardial infarction in sheep hearts. Development of the chronic disease models has been described elsewhere for each chronic cardiac disease model, myocardial infarction induced by percutaneous coronary artery embolization ${ }^{13}$ and self-sustaining atrial fibrillation ${ }^{30}$.

\section{Protocol}

All experiments were performed following the guidelines from Directive 2010/63/EU of the European Parliament on the protection of animals used for scientific purposes. Animal protocols were approved by the local ethical committee (CEEA50) at the University of Bordeaux. Hearts were sourced from three large mammalian models, including (i) Healthy Large white pigs ( $N=2,2$ months old); (ii) Sheep $(N=1,2$ years old) with induced myocardial infarction ${ }^{13}$ and (iii) Sheep $(\mathrm{N}=1,7$ years old $)$ with induced atrial fibrillation ${ }^{30}$.

\section{Solution preparation:}

1. Cardioplegic solution: Prepare $3 \mathrm{~L}$ of distilled water and add sodium chloride (110 mM), potassium chloride (16 $\mathrm{mM})$, Sodium bicarbonate (10 mM), D-(+)-Glucose (9 $\mathrm{mM}$ ), calcium chloride solution (1.2 mM) and magnesium chloride solution $(16 \mathrm{mM})$. At the end, add $500 \mu \mathrm{L} / \mathrm{L}$ of heparin sodium. Conserve this solution at $4{ }^{\circ} \mathrm{C}$.

2. Phosphate buffered saline - EDTA solution (PBS-EDTA).

1. First, add ethylenediaminetetraacetic acid (EDTA) to $1 \mathrm{~L}$ of distilled water for a final concentration of $10 \mathrm{mM}$. Increase and maintain a solution $\mathrm{pH}$ of 12 using sodium hydroxide solution (1 $\mathrm{M})$ to dissolve the EDTA.

2. Once the EDTA is fully dissolved, lower the $\mathrm{pH}$ to 7.4 using hydrochloric acid. Add one foil pouch of phosphate-buffered saline to obtain a solution at $0.01 \mathrm{M}$ (sodium chloride, $0.138 \mathrm{M}$; potassium chloride, $0.0027 \mathrm{M}$ ) and $\mathrm{pH}$ 7.4. Conserve this solution at room temperature (RT).

3. Ethanol - phosphomolybdic acid (PMA) contrast agent solution: Prepare $1 \mathrm{~L}$ of absolute ethanol and add the PMA to obtain a solution at $1 \%$ of concentration. Conserve this solution at RT.

\section{Source of tissue}

1. Euthanize the animal and extract the heart according to local ethical guidelines. Quickly immerse the heart into cold cardioplegic solution and gently massage the ventricles for initial rinsing.

2. Ensure to cut the aorta below the aortic arch and clamp two sides of the arterial wall using needle holders.

3. Suspending the heart by the needle holders, insert an aortic cannula into the aortic root, taking care not to make contact with or protrude through the aortic valves. Wrap a 0 gauge suture around the aortic arch at the level of the cannula and firmly tie the cannula in place.

4. Using $50 \mathrm{~mL}$ syringes, inject $200 \mathrm{~mL}$ of cold $\left(4{ }^{\circ} \mathrm{C}\right)$ cardioplegic solution. Remove excess blood pooling in the cavities by tipping the heart on its posterior side to drain via the pulmonary veins.

5. Immerse the rinsed heart and keep in cold cardioplegic solution stored on ice until ready for dissection.

\section{Tissue preparation:}

1. Prepare a $1 \mathrm{~L}$ reservoir supported $80 \mathrm{~cm}$ above a dissection dish. Couple a thermoplastic tube $80 \mathrm{~cm}$ in length and $3.2 \mathrm{~mm}$ internal diameter and $4.8 \mathrm{~mm}$ external diameter to a drain port of the reservoir. 
2. Fix a three-way tap to the drainage tubing and couple further thermoplastic tubing $(20 \mathrm{~cm}, 1.6 \mathrm{~mm}$ internal diameter and $3.2 \mathrm{~mm}$ external diameter) to each free port on the three-way tap. Fix two-way taps to the fee ends of the tubing.

3. Fill the reservoir with the cardioplegic solution supplemented with heparin (2500 units). Open the taps to allow the cardioplegic solution to drain and remove all air bubbles, then close the two-way taps.

4. Prepare cannulae for left and right coronary ostia using Polytetrafluoroethylene (PTFE) tubing (1 $\mathrm{mm}$ internal diameter and $2 \mathrm{~mm}$ outer diameter).

1. Cut $5 \mathrm{~cm}$ of tubing and heat one end by placing the tip next to a naked flame. Once $1 \mathrm{~mm}$ of the tip begins to melt and becomes translucent, press the tip against a hard heat resistant surface to shape a ridge at the cannulae tip to prevent cannulae from slipping out of the vessels.

2. Insert $1 \mathrm{~cm}$ of the non-heated end of each cannula in to the two ends of the drain reservoir drainage tubing.

5. Remove the aortic cannula. Under cold cardioplegic solution, localize the left and right ostia of coronary arteries.

6. Using pointed scissors, carefully separate the aortic root from the surrounding tissue above and below the coronary ostia to enable threading of a $0 \mathrm{G}$ silk suture under the coronary vessel.

7. Open the two-way taps and insert the cannulae tips into the coronary ostia. With the cannulae tips extending 1-2 $\mathrm{cm}$ into the ostia and beyond the suture placement, tie off cannulae.
8. Rinse the heart while gently massaging the ventricles for 15 min until the heart is cleared of blood.

9. After rinsing, close the two-way taps and disconnect them from the three-way tap. Transfer the heart to a $1 \mathrm{~L}$ plastic chemical-resistant container containing $500 \mathrm{~mL}$ of PBS-EDTA solution.

10. Recirculate PBS-EDTA solution in the thermoplastic tubing under a fume hood using a peristaltic pump with two channels. Prime the pump tubing until the tubing is absent of air bubbles, then perfuse each coronary artery cannulae by recirculation at $\mathrm{RT}$ for $2 \mathrm{~h}$ at $80 \mathrm{~mL} / \mathrm{min}$.

11. Ensure that the fume hood is operational. Stop the pump, drain the solution from the container and replace it with formalin (10\%) for fixation for $1 \mathrm{~h}$ at $\mathrm{RT}$ at $80 \mathrm{~mL} / \mathrm{min}$.

12. Replace the formalin solution with PBS to rinse the fixative three times for $15 \mathrm{~min}$ each at $80 \mathrm{~mL} / \mathrm{min}$.

\section{Tissue dehydration and drying:}

NOTE: Use the same perfusion rate $(80 \mathrm{~mL} / \mathrm{min})$ and let the tissue remain at $\mathrm{RT}$ throughout.

1. Replace PBS solution with ethanol at $20 \%$, diluted in ultra-pure water, and perfuse for a minimum of $3 \mathrm{~h}$.

2. Perfuse the heart using a series of incrementing ethanol concentrations.

1. Start by replacing the $20 \%$ ethanol solution with ethanol diluted to $30 \%$ and perfuse for $2 \mathrm{~h}$.

2. Repeat perfusion by incrementing the ethanol concentration at each iteration through $40 \%, 50 \%$, $60 \%, 65 \%, 70 \%, 75 \%, 80 \%, 95 \%, 90 \%, 95 \%, 99 \%$, and $100 \%$ for a minimum duration of $1 \mathrm{~h}$ at each step(concentration). 
NOTE: Heart samples may rest with no perfusion flow overnight at any ethanol dilution if minimum perfusion of $15 \mathrm{~min}$ has taken place for that concentration.

3. OPTIONAL: If applying contrast agents via perfusion, perfuse the heart with $100 \%$ ethanol supplemented with the contrast agent PMA, $1 \%$ for $48 \mathrm{~h}$. Rinse the contrast agent by perfusion with $100 \%$ ethanol for $2 \mathrm{~h}$.

4. To reinforce the heart tissue prior to air drying, recirculate a 50:50 mix of ethanol and hexamethyldisilazane (HMDS) for $10 \mathrm{~min}$. Follow this by $100 \%$ HMDS for a further $2 \mathrm{~h}$.

CAUTION: HMDS is a highly toxic and noxious substance. A strong odor of ammonia is released in contact with air. Moreover, the liquid form of HMDS is highly volatile and catalyzed by iodine-containing agents.

5. Disconnect the cannulae from the tubing and suspend the heart from an aortic suture inside the fume hood.

6. Carefully slide a zip-lock bag over the heart and close the bag seal over the suture to reduce exposure of the heart to circulating air. Allow the heart to dry through evaporation for 1 week.

7. OPTIONAL: For diffusion-loading contrast agents, wash the heart in $100 \%$ ethanol for 15 min while agitating. Immerse the heart in $100 \%$ ethanol supplemented with PMA, $1 \%$, for $48 \mathrm{~h}$ under vacuum. Repeat step 4.6.

\section{MicroCT:}

NOTE: A desktop X-ray microCT system was used for imaging pig hearts.

1. Mount the air-dried heart onto an appropriate sample holder. Prevent any movement during the $\mathrm{X}$-ray microCT measurements using a clamp anchored to the sample holder and secure the heart via the dried and rigid aorta.

2. Meticulously align the center of the heart sample along its longitudinal axis with the center of the imaging field of view for $0^{\circ}$ and $90^{\circ}$ angles of rotation. To achieve this in all orientations, suspend the heart in the air via an aortic clamp fixed to the sample support.

3. After opening the software and initiating the X-ray microCT system, apply the X-ray filter aluminum, $1 \mathrm{~mm}$, $\mathrm{X}$-ray source voltage to $60 \mathrm{kV}$ and current to $120 \mu \mathrm{A}$. Set image dimensions to 2016 × 1344 pixels and pixel size to $20 \mu \mathrm{m}$.

4. Retract the sample holder out of the field of view and calibrate the background image and X-ray exposure time by obtaining a flat-field correction. Ensure that the average background $\mathrm{X}$-ray transmission is greater than $80 \%$.

5. Scout X-ray transmission images along the length of the support to determine the overall imaging field in the heart's longitudinal axis. For scanning, use a rotation step of $0.18^{\circ}$, a frame averaging of 5 , and a sample rotation of $180^{\circ}$. Select the offset scanning mode to image the full width of the sample support.

NOTE: The acquisition parameters indicated in this section have been selected to optimize the image quality of the ensemble heart composition.

6. After scanning, use the software for tomographic reconstruction of an isotropic three-dimensional image volume. For the application of NRecon software, use acquisition-related artifact correction, including beamhardening effects of $10 \%$ and ring artifact reduction of 8 .

7. To optimize data storage limitations, apply the minimum rectangular region of interest that encompasses heart- 
specific image voxels. Export the images in an 8-bit bitmap format as an image stack.

8. Visualize the reconstructed data stack using DataViewer software. Digitally orientate the sample within the image boundaries to realign the sample's long and short axes with the three principal axes of the image volume.

9. Crop the image volume in all three axes to remove outer background layers of the image, to maximally reduce the total image size.

\section{Representative Results}

The preparation of large mammalian hearts using the dehydration and air-drying method removes all water content from the sample. Evidence of insufficient water replacement by ethanol can be observed during HMDS loading (see Protocol, step 4.4). The presence of water under HMDS will create bubbles rising from the tissue. In the case of excessive water levels, a rise in the temperature of the immersion fluid can occur. Keeping the immersion chamber surrounded by ice during initial HMDS loading can reduce the ill-effects of tissue heating. After air-drying hearts in the absence of contrast agents, the sample will appear white in color (see Protocol, step 4.6). The outer surface was often dried and structurally stable before intramural layers. Rinsing in ethanol prior to contrast agent loading removed the white deposit (see Protocol, step 4.7). Slicing through tissue using a sharp blade reveals macroscopically individual muscle fibers with clear separation. Contrast loading by immersing heart samples in contrast agent medium suffered from diffusion limit artifacts in thick and highly muscular regions of the sample. Diffusion contrast loading under vacuum provided more homogeneous coloration in muscle (heart sample \#1, see Table 1 for contrast agent loading times). Macroscopically, the surface contrast agent distribution showed in-homogeneous staining between heart muscle and regions composed primarily of extracellular components, notably fat and connective tissue. Air-dried tissue samples, either prior to or after contrast agent loading, maintained stable structural integrity.

The time necessary to scan the full width of the sample at $20 \mu \mathrm{m}$ resolution under microCT using the abovementioned scanning parameters and an exposure time of 1700 ms was $6 \mathrm{~h} 34 \mathrm{~min}$. Depending on the size of the sample in the gantry axis of the scanner, this duration was multiplied by the number of positions needed to capture the full length of the specimen. For pig and sheep hearts in this study, three to four positions were used. The NRecon software tiled the multi-position and offset scans to form a single X-ray projection image for each rotation step of the X-ray source and detector. In total, 1000 projections are stored as 16-bit images, generating 30-40 GB of data. Reconstructed volumetric images were 52-70 GB.

Major anatomical landmarks, including the ventricular cavities, septum, and free walls of the ventricles, were easily identifiable from X-ray transmission imaging of airdried pig hearts stained with contrast agent by diffusionloading (Figure 1A). Moreover, highly textured regions indicating microstructural organization, such as myocardial fiber orientation, were also observed due to sensitive X-ray attenuation/transmission (Figure 1B). Tomographic reconstructions of three-dimensional image volumes showed distinct separation between tissue and background at both epicardial and endothelial boundaries (Figure 1D). Intramurally, low contrast and voxel intensity diffusion gradient were observed throughout thick transmural regions of the tissue. Despite that, vasculature and myocardial fibers separated by cleavage planes were still readily identifiable. $A$ second higher intensity bandwidth of contrast was observed at the epicardial-most layer and in punctate sub-endocardial 
regions. Contrast enhancement was greatest at sites where extracellular components were accumulated, particularly epicardial connective tissue, epicardial fat and the connective tissue sheath of the Purkinje fiber network. Voxel signal intensity distributions showed high separation from the zerointensity background (air) and two dominant populations of low and high contrast tissue (Figure 1D).

To validate contrast enhancement of microCT image reconstructions and the selectivity to collagenous compartments of the heart samples, histology, bright field microscopy, and fluorescent microscopy were employed (Figure 2). A transmural block of ventricular tissue from an air-dried heart without prior contrast agent-loading was prepared for paraffin embedding and sectioning. Adjacent tissue slices mounted on microscope slides were treated by either Masson's trichrome staining, no treatment, or $48 \mathrm{~h}$ of PMA (1\%). Immersion of slide-mounted tissue sections eliminated diffusion gradient effects of the staining process that was observed in whole heart samples. Mason's trichrome staining showed collagen-positive staining at the epithelial and endothelial layers, perivascularly in the sub-epicardial tissue, and a connective tissue sheath surrounding a freerunning Purkinje fiber protruding into the left ventricular cavity (Figure 2A). Bright field illumination showed darker coloration in collagenous structures after PMA-staining, supporting the preferential accumulation of PMA (Figures 2B,C). Moreover, PMA treatment has previously been shown to quench the autofluorescence of collagen macromolecular complexes ${ }^{31}$. Fluorescent images of ventricular tissue sections had PMAinduced loss of fluorescence at sites of collagen (Figure 2D vs. $2 E$, Figure $2 D^{\prime}$ vs. $2 E^{\prime}$ and Figure $2 D^{\prime \prime}$ vs. $\left.2 E^{\prime \prime}\right)$. In both bright field and fluorescent imaging, cellular compartments were not altered by the PMA treatment, and collagen had a selective accumulation of PMA staining and quenching of autofluorescence.

Heart sample \#2 was stained with a contrast agent via perfusion prior to air drying. Image reconstruction revealed highly patchy staining within the myocardial compartment (Figure 3A). Contrast enhancement appeared unselective of tissue composition, with no further enhancement of signal intensity at the epicardial or sub-endocardial regions. Moreover, low contrast tissue showed poor separation from the background intensity (Figure 3B).

Ventricular fibrosis was induced by myocardial infarction and chronic ischemia (Heart sample \#3). An antero-apical scar was formed by replacing myocytes with fibro-fatty deposits in the tissue downstream to the site of vascular embolization. Heart sample \#3 was prepared and imaged from a dissected ventricular wedge covering the anterior left ventricle, septum, and right ventricular free wall. The preparation of this ventricular wedge configuration has been described previously ${ }^{32}$ and the application of wedges for cardiac imaging was reviewed in detail ${ }^{33}$. Scar morphology was transmural but heterogeneous (Figure 4). A central dense fibrotic lesion was surrounded by a loose and heterogeneous border zone (Figure 4A). The ventricular preparation was stained by diffusion-loading post-air drying and in a vacuum. Figure 4B-E shows the greatest signal intensities of reconstructed microCT image volumes at the tissue boundaries and scar regions. Contrast agents poorly stained healthy myocardium, yet microstructural contrast was retained (Figure $\mathbf{4 C}^{\prime}$ ). At the border zone, scar tissue was interspersed with surviving myocardium (Figure 4D'). Dense fibrosis appeared transmural yet textured, indicating variances in composition (Figure 4E'). Tissue sections of a transmural left ventricular region of the air-dried and 
PMA-stained tissue preparation were used to validate PMA selectivity for collagen in pathological tissue by comparing against Masson's trichrome staining (Figure 4F). PMA staining was selective for collagen (sub-epicardium and subendocardium) and absent in regions of surviving myocardium (Figure 4G).

Heart sample \#4 with induced persistent atrial fibrillation was air-dried while conserving the native shape of the atrial cavity. Atrial appendage collapse was not observed. The major anatomical landmarks could be identified morphologically from reconstructed images (the atrial septum, pectinate muscles, coronary sinus, pulmonary vein ostia, vena cava and cristae terminalis). Diffusion-staining under vacuum resulted in contrast enhancement in the aortic root and atrioventricular valves and discrete regions of the working myocardium. Muscle staining enhancement was constrained to the atrial appendages and posterior walls of both left and right atria (Figure 5).
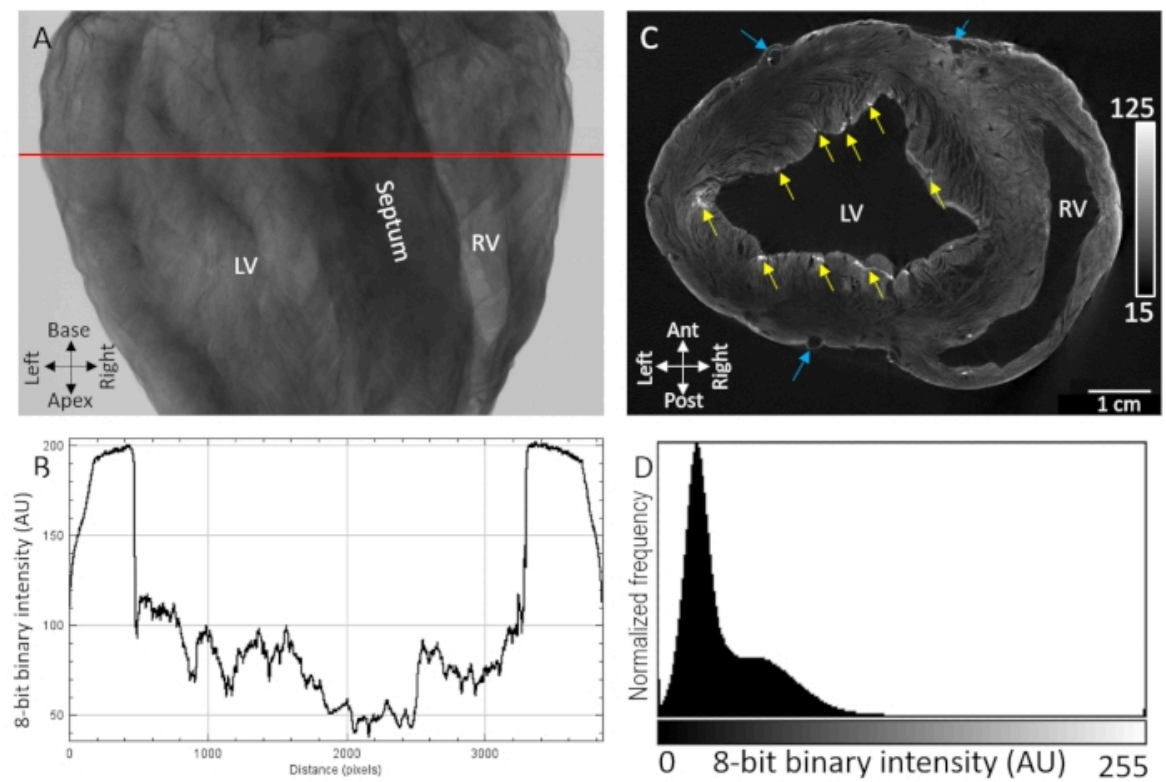

Figure 1: MicroCT imaging of an air-dried pig heart treated with PMA contrast agent by diffusion under vacuum. (A) X-ray projection image. (B) A transmission profile extracted from the red line in A. (C) Short-axis slice of the ventricles from a tomographically reconstructed three-dimensional volume. Yellow arrows indicate punctate regions of contrast attributed to sub-endocardial Purkinje fibers. Blue arrows indicate vasculature. (D) Signal intensity distribution of the reconstructed image slice shown in C. LV: left ventricle and RV: right ventricle. Please click here to view a larger version of this figure. 

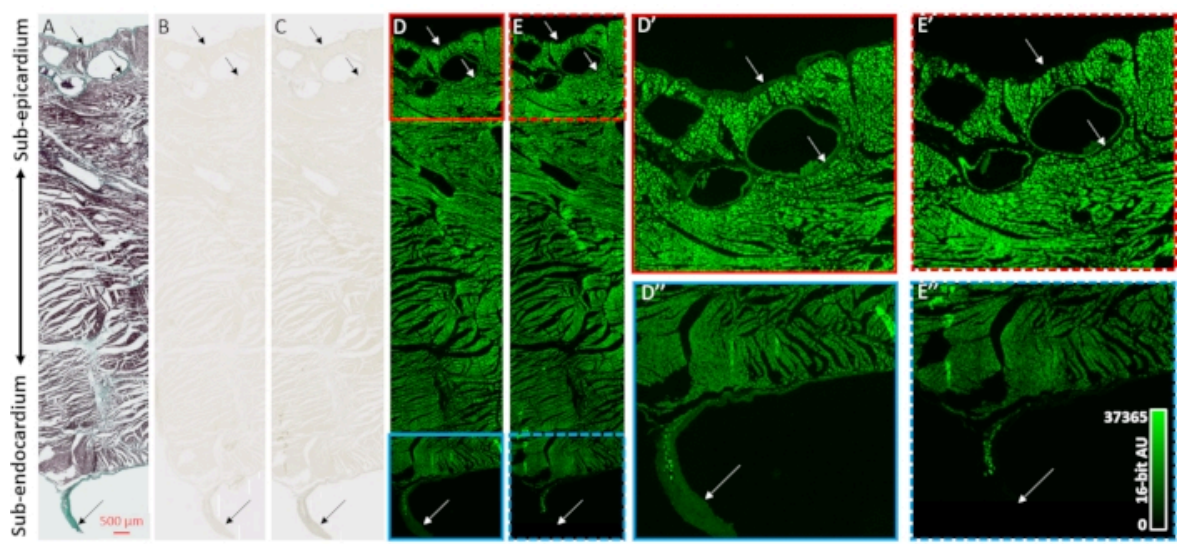

Figure 2: Validation of PMA-selectivity for collagen. (A) Masson's trichrome staining of a transmural tissue section from the ventricles of an air-dried heart. Myocardium is stained in red and collagen is shown with green coloration. Adjacent tissue sections (B) absent of staining or (C) stained with PMA (1\%) were imaged with bright field illumination to assess the uniformity of coloration. (D) Tissue sections absent of staining or (E) stained by PMA were imaged by fluorescent microscopy. Panels D' (solid red box) and E' (dashed red box) are enlarged views of the sub-epicardium for unstained and PMA-stained sections. Panels D" (solid blue box) and E" (dashed blue box) are corresponding enlarged views of the subendocardium and a free-running Purkinje fiber. Arrows indicate sites of collagen content. Please click here to view a larger version of this figure. 

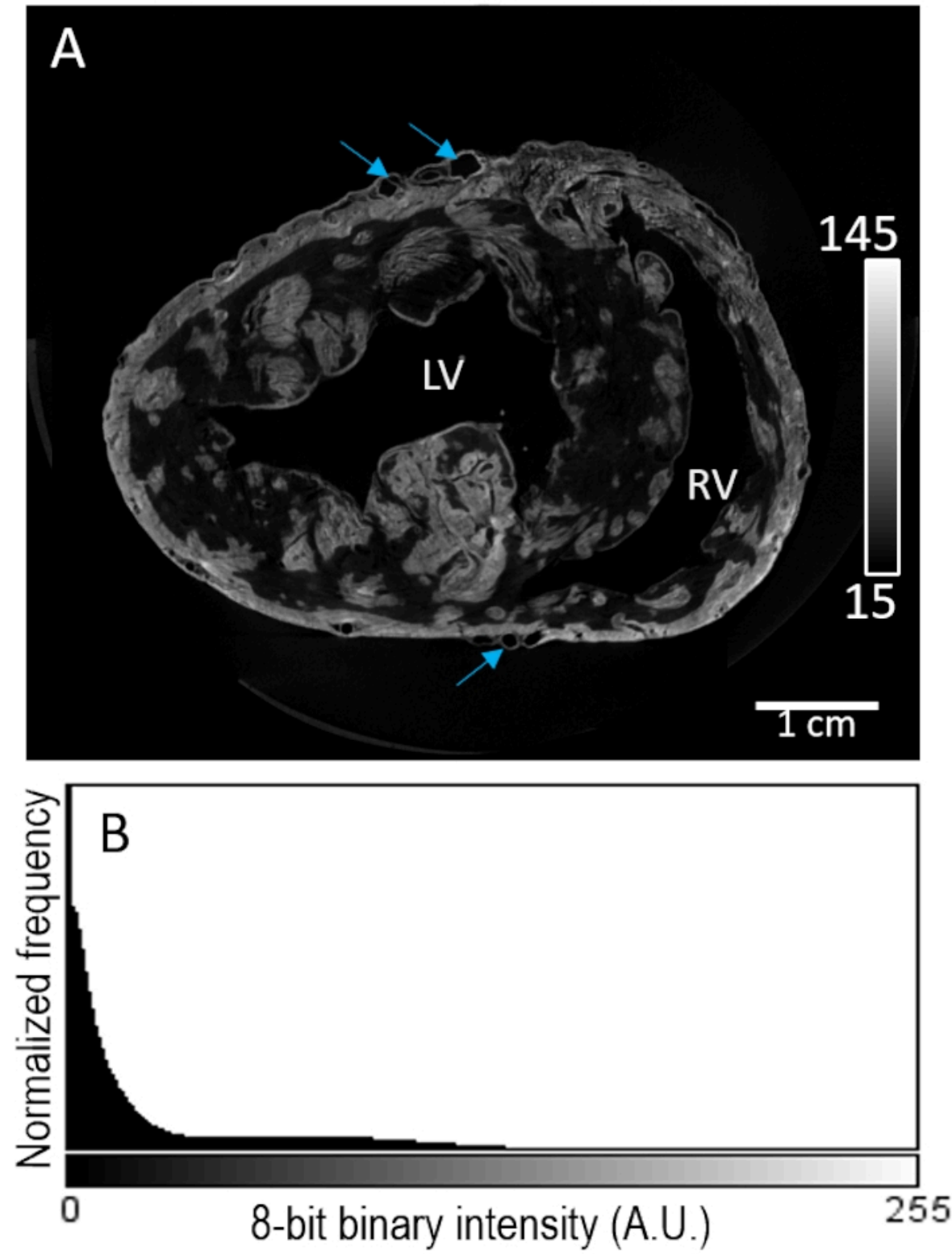

Figure 3: Perfusion-loading of PMA prior to air-drying and MicroCT imaging. (A) A short-axis slice of a reconstructed image volume of the ventricles from a pig heart. Blue arrows indicate vasculature. (B) The signal intensity distribution of the image slice from panel A. LV: left ventricle and RV: right ventricle. Please click here to view a larger version of this figure. 

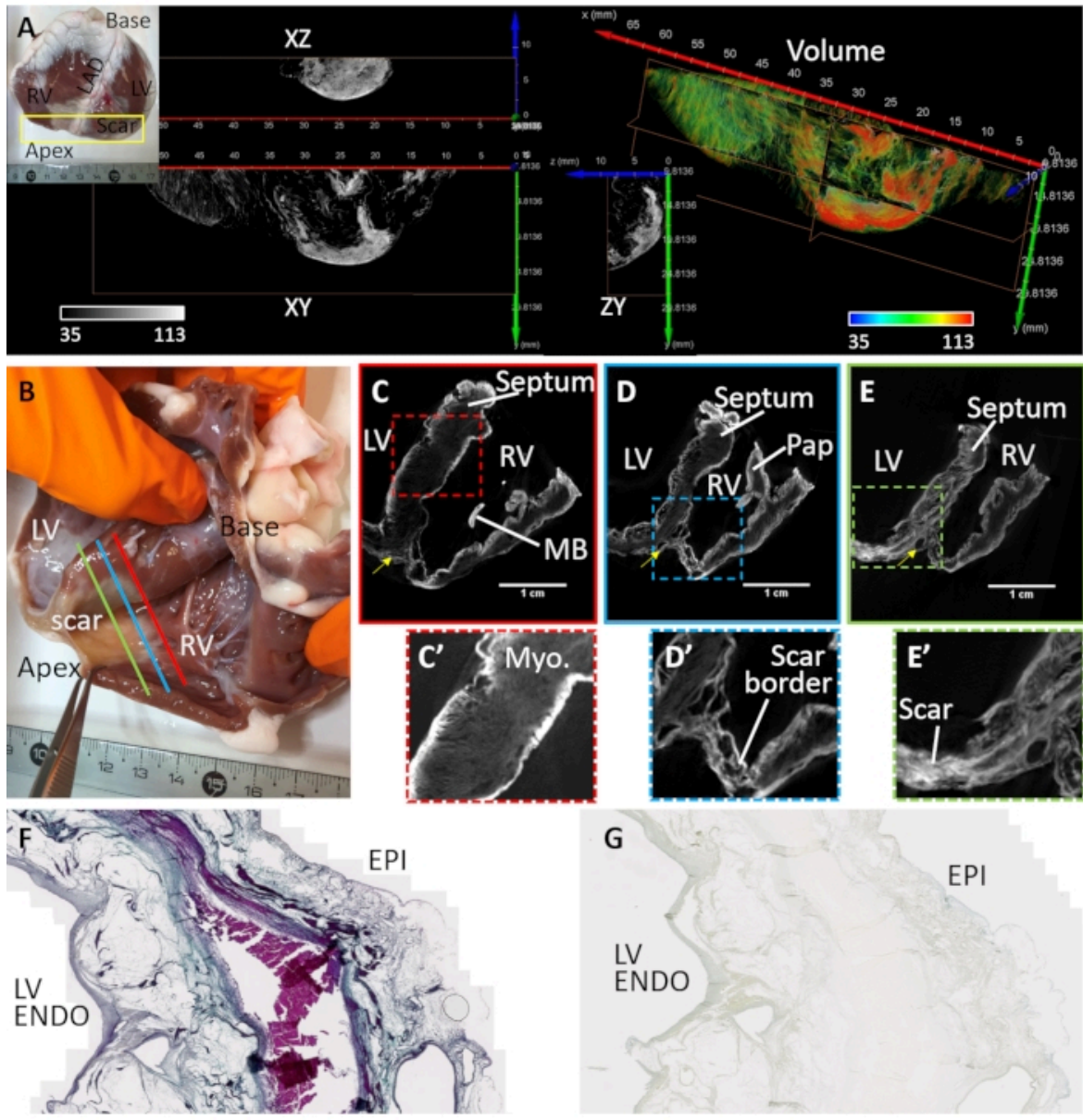

G

EPI

LV

ENDO

Figure 4: MicroCT imaging of a sheep heart suffering a chronic myocardial infarction. (A) A dense scar was formed in the apical region (see inset photograph). A volume rendering of the apical region from an endocardial perspective was assigned coloration based on image intensity (red corresponding to scar tissue and myocardium in green). Orthogonal slices of the greyscale intensity show the dense scar distribution and bordering surviving myocardium. Separation between fibrotic tissue and myocardium corresponds to regions of adipose tissue. (B) A photograph of an air-dried ventricular wedge preparation from a sheep with apical scarring following myocardial infarction. Oblique slices of the reconstructed microCT image volume traverse the ventricles at the mid-level between base and apex and proximal to the site of (C) vascular occlusion (C- red line in panel B), (D) the peri-infarct region bordering dense scar and healthy myocardium (D-blue line in panel $\mathbf{B})$ and $(\mathbf{E})$ a region of dense fibrosis (E - green line in panel $\mathbf{B})$. (C') An expanded view of the septal region outlined by a red dashed box in C. (D') An expanded view of the infarct region in the right ventricular apex (blue dashed box in panel D). (E') An expanded view of the infarct region in the left ventricular apex (green dashed box in panel E). LV: Left ventricular cavity; RV: right ventricular cavity; MB: moderator band; Pap: papillary muscle. Yellow arrow indicates left anterior descending artery. (F) Masson's trichrome staining of a histological section cut from the PMA-stained air-dried left ventricle. 
Collagen is stained blue and myocardium is stained pink/violet. (G) A corresponding tissue section of the PMA-staining distribution. Please click here to view a larger version of this figure.
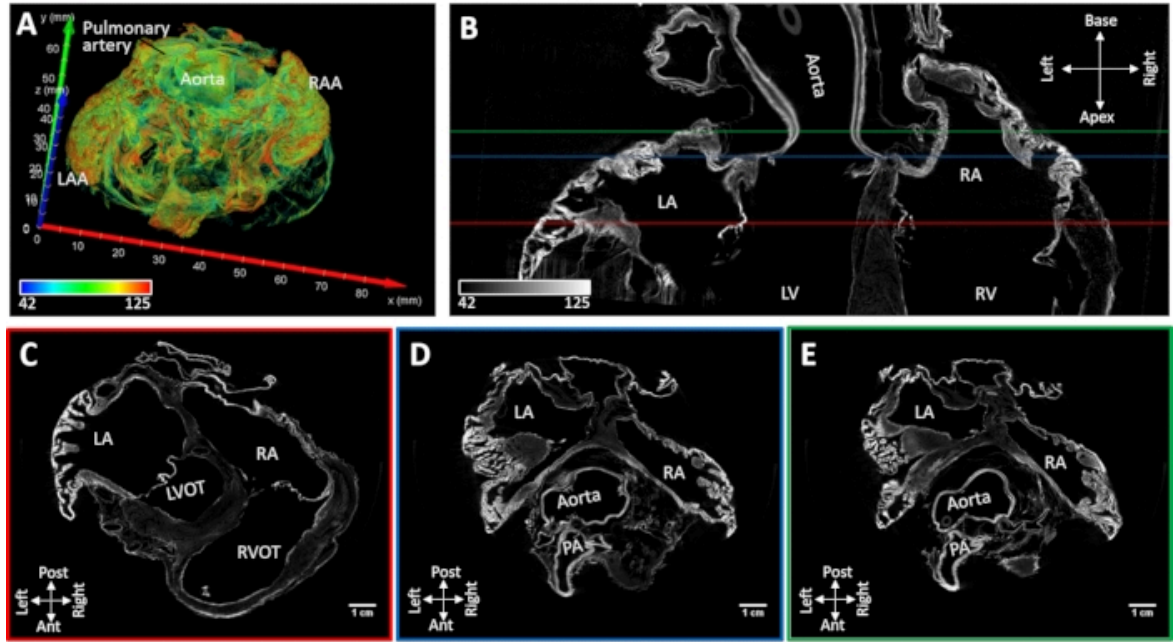

Figure 5: MicroCT image of a sheep heart following chronic induced atrial fibrillation. (A) A volume rendering of the atria with coloration assigned as in Figure 4A. (B) Bi-atrial microCT image slice in the long-axis of the heart. Short-axis slices were extracted at the level of the (C) atrioventricular valves (C- red line in panel B), (D) aortic root (D- blue line in panel B) and $(E)$ left atrial roof (E- green line inpanel B). LA: left atria; RA: right atria; LAA: left atrial appendage; RAA: right atrial appendage; LV: left ventricle; RV: right ventricle; LVOT: left ventricular outflow tract; RVOT: right ventricular outflow tract and PA: pulmonary artery. Please click here to view a larger version of this figure. 


\begin{tabular}{|c|c|c|c|c|}
\hline Sample \# & $\mathbf{1}$ & $\mathbf{2}$ & $\mathbf{3}$ & $\mathbf{4}$ \\
\hline Species & Pig & Pig & Sheep & Sheep \\
\hline Body weight (kg) & 32.4 & 31.2 & 202.4 & 207.6 \\
\hline Heart weight (g) & 191.2 & 186.2 & Chronic Ml & Chronic AF \\
\hline Pathology & - & - & Wedge of anterior heart & Whole heart \\
\hline Sample preparation & Whole heart & Whole heart & Diffusion & Diffusion \\
\hline $\begin{array}{c}\text { Mode of } \\
\text { contrast loading }\end{array}$ & Diffusion & Perfusion & & 48 \\
\hline $\begin{array}{c}\text { Contrast agent } \\
\text { exposure (h) }\end{array}$ & 48 & 24 & & \\
\hline
\end{tabular}

Table 1: Heart samples and contrast agent treatment.

\section{Discussion}

A detailed protocol for large tissue preparations is set out using whole hearts from large mammals for subsequent high-resolution structural imaging. An air-drying approach removed influences of background X-ray attenuation and maximally optimizing tissue: background contrast ${ }^{29}$. Using this approach, an isotropic resolution in the range of $20 \mu \mathrm{m}$ for volumetric imaging across samples up to $7.2 \mathrm{~cm}$ in diameter was achieved. MicroCT of soft tissue however typically relies on the use of non-specific contrast agents to ameliorate X-ray absorption and sensitivity of microCT systems ${ }^{34}$. Although X-ray contrast agents improve overall X-ray attenuation and soft tissue imaging enhancement, separation of tissue constituents based on biochemical composition remains challenging. However, it was observed that using air-dried hearts in combination with a common X-ray contrast agent in the laboratory setting, PMA, selectively stained extracellular components. Connective tissue associated with healthy myocardium and pathological structural remodeling in chronic diseases were enhanced.

The process of air-drying biological tissue demands an intervention to resist the deformation of the sample. Sample preparation for electron microscopy has similar requirements. Typically, a critical-point drying method is employed, which uses a balance of tissue immersion medium, temperature, and pressure to eliminate surface tension of the tissue's liquid content, which causes deformation at the molecular level upon evaporation ${ }^{35}$. This approach requires uniform replacement of the sample's water content with liquid carbon dioxide, which is more reliable in small and easily diffusible samples. Alternatively, the structural integrity of the tissue can be improved and air-drying, i.e., the evaporation phase can be applied over a longer period to reduce overall deformation. The molecule HMDS undergoes silylation to form a silicone-based scaffold to reinforce and stabilize the molecular organization of the tissue sample ${ }^{36}$. The evaporation is further prolonged by limiting circulating air 
currents from the environment, also to avoid inhomogeneous evaporation, particularly between the sample surface and intramural layers.

Numerous contrast agents have previously been used for microCT imaging of soft tissues. The most common are iodine, phosphotungstic acid (PTA) and PMA. lodine particularly has been employed due to a higher diffusion rate $34,37,38$. Nevertheless, iodine acts as a catalyst for the silylation of HMDS reagent ${ }^{36}$. The catalyzed reaction is aggressive and exothermic, with a high risk of the destruction of the specimen and safety risk if residual HMDS remains due to incomplete desiccation of the sample. Both PTA and PMA dissolved in ethanol can safely be used in conjunction with HMDS. PTA and PMA have been shown to provide greater resolving power of fine structures in nonmineralized intervertebral discs when compared to iodine staining $^{38}$. In microCT imaging of mammalian samples, PTA and PMA have been used for staining mouse embryos ${ }^{39}$, mouse cardiovascular system ${ }^{37}$, rabbit muscle and brain ${ }^{40}$, and porcine veins ${ }^{41}$. PTA has a higher molecular mass and density in solution than PMA. This is partly due to a higher atomic mass of tungsten (atomic number is $74 \mathrm{~g} / \mathrm{mol}$ ), the principal attenuating element in PTA. By comparison, the heaviest element in PMA, molybdenum, has an atomic number of $42 \mathrm{~g} / \mathrm{mol}$. Both atomic mass and sample density underlie X-ray attenuation, in addition to the sample thickness ${ }^{42}$. Increasing the $\mathrm{X}$-ray path length by augmenting sample sizes, X-ray attenuation becomes more sensitive to increased sample density. Therefore, the lower density PMA contrast agent was selected to reduce the risk of over attenuation and to optimize the dynamic range of image contrast for hearts of human-like scale. Further evidence has shown that diffusion-loading of PMA gives more homogeneous staining than for the larger molecule PTA in cardiac tissue $^{43}$.

The method of contrast agent delivery impacts the uniformity of contrast agent distribution in heart tissue (Figure 3). Perfusion of contrast agents in the ethanol-dehydrated heart showed patchy background staining levels of PMA due to variable vascular resistance. In the air-dried heart, the muscle laminar structure is emphasized by the sample desiccation process, increasing muscle laminar separation. This ultimately improved the overall permeability of the tissue for diffusion-based contrast agent loading. Consequently, airdrying facilitated tissue: air contrast at the laminar and intralaminar levels (Figure 4). Moreover, diffusion-loading can be further facilitated by application under a vacuum. It has further been shown that tissue shrinkage of non-dried samples is dependent upon contrast agent concentration ${ }^{40}$. However, prior morphological stabilization of the specimen by air-drying inhibits tissue shrinkage effects ${ }^{29}$.

High-resolution microCT images of whole organs inherently produce large data volumes. The nature of tomographic imaging techniques enables visualization and image handling on a slice-by-slice basis, which eases the computer processing and memory burden. However, to visualize three-dimensional image stacks, for example, to render specimen volumes in three-dimensional representations, the recommended minimum computer specifications are $128 \mathrm{~GB}$ RAM and a processor speed of $3 \mathrm{GHz}$. Solid-state hard drives also greatly improved data transfer.

The emergence of microCT imaging in the cardiac field affords numerous advantages for translational studies and clinical validation. The advantages of its threedimensional and micrometric imaging have already shown applications in determining the thrombotic burden of 
ST-elevation myocardial ischemia patients ${ }^{44,45}$. Mapping potential sources of arrhythmia in structural heart disease patients is largely dependent on determining the distribution of fibrotic scar tissue and localizing interweaving tracks of surviving myocardium. Second-line approaches for diagnosis of ventricular arrhythmias utilize magnetic resonance imaging $^{46}$. It can robustly localize dense fibrosis but is limited to low-resolution morphological characterization and offers limited insight into microstructural remodeling and diffuse distributions of fibrotic lesions ${ }^{47}$. High-resolution examination of scar distribution and characterization has vast potential for improving our understanding of cardiac structural remodeling and the risk of developing heart failure. Particularly, fundamental research studies or post-mortem investigations will benefit from corroborative structural images for electrical mapping of cardiac arrhythmia.

In conclusion, hearts reinforced with HMDS treatment and airdrying can subsequently be stained with an X-ray contrast agent to enhance the X-ray attenuation of extracellular components. Specifically, in healthy myocardium, PMA accumulation occurs at the epithelium, valvular tissue, and compartments of the ventricular conduction system sheathed by connective tissue resulted in enhanced $\mathrm{X}$-ray attenuation. Moreover, in structurally diseased myocardium, enhanced contrast was further selective for fibrosis.

\section{Disclosures}

None

\section{Acknowledgments}

This study received financial support from the French Government as part of the "Investments of the Future" program managed by the National Research Agency (ANR), Grant reference ANR-10-IAHU-04, and the Leducq
Foundation (RHYTHM network), as well as Grant reference ANR-17-CE14-0029-01 [UNMASC], funding from the European Research Area in Cardiovascular Diseases (ERACVD), grant reference H2020-HCO-2015_680969 [MultiFib] and funding from the French Region Nouvelle Aquitaine, grant references 2016 - 1R 301130000 7550/2016-1R 301130000 7553 and ANR-19-ECVD-0006-01.

\section{References}

1. Srinivasan, N. T., Schilling, R. J. Sudden cardiac death and arrhythmias. Arrhythmia \& Electrophysiology Review. 7 (2), 111-117 (2018).

2. Szumowski, L. et al. Mapping and ablation of polymorphic ventricular tachycardia after myocardial infarction. Journal of the American College of Cardiology. 44 (8), 1700-1706 (2004).

3. Bode, K. et al. Ablation of polymorphic ventricular tachycardias in patients with structural heart disease. PACE - Pacing and Clinical Electrophysiology. 31 (12), 1585-1591 (2008).

4. Enjoji, Y. et al. Catheter ablation of fatal ventricular tachyarrhythmias storm in acute coronary syndromerole of Purkinje fiber network. Journal of Interventional Cardiac Electrophysiology. 26 (3), 207-215 (2009).

5. Sinha, A. M. et al. Role of left ventricular scar and purkinje-like potentials during mapping and ablation of ventricular fibrillation in dilated cardiomyopathy. PACE Pacing and Clinical Electrophysiology. 32 (3), 286-290 (2009).

6. Peichl, P., Čihák, R., Koželuhová, M., Wichterle, D., Vančura, V., Kautzner, J. Catheter ablation of arrhythmic storm triggered by monomorphic ectopic beats in patients 
with coronary artery disease. Journal of Interventional Cardiac Electrophysiology. 27 (1), 51-59 (2010).

7. Marrouche, N. F. et al. Mode of initiation and ablation of ventricular fibrillation storms in patients with ischemic cardiomyopathy. Journal of the American College of Cardiology. 43 (9), 1715-1720 (2004).

8. Bänsch, D. et al. Successful catheter ablation of electrical storm after myocardial infarction. Circulation. 108 (24), 3011-3016 (2003).

9. Yokoshiki, H., Mitsuyama, H., Watanabe, M., Mizukami, K., Tsutsui, H. Suppression of ventricular fibrillation by electrical modification of the Purkinje system in hypertrophic cardiomyopathy. Heart and Vessels. 29 (5), 709-717 (2014).

10. Agress, C. M., Rosenberg, M. J., Jacobs, H. I., Binder, M. J., Schneiderman, A., Clark, W. G. Protracted shock in the closed-chest dog following coronary embolization with graded microspheres. The American journal of physiology. 170 (3), 536-549 (1952).

11. Bolukoglu, H., Liedtke, A. J., Nellis, S. H., Eggleston, A. M., Subramanian, R., Renstrom, B. An animal model of chronic coronary stenosis resulting in hibernating myocardium. American Journal of Physiology - Heart and Circulatory Physiology. 263 (1 Pt 2), H20-29 (1992).

12. Capone, R. J., Most, A. S., Sydlik, P. A. Precordial ST segment mapping. A sensitive technique for the evaluation of myocardial injury? CHEST. 67 (5), 577-582 (1975).

13. Dib, N., Diethrich, E. B., Campbell, A., Gahremanpour, A., McGarry, M., Opie, S. R. A percutaneous swine model of myocardial infarction. Journal of Pharmacological and Toxicological Methods. 53 (3), 256-263 (2006).
14. Dogné, J. M. et al. Characterization of an original model of myocardial infarction provoked by coronary artery thrombosis induced by ferric chloride in pig. Thrombosis Research. 116 (5), 431-442 (2005).

15. Eldar, M., Ohad, D., Bor, A., Varda-Bloom, N., Swanson, D. K., Battler, A. A closed-chest pig model of sustained ventricular tachycardia. Pacing and Clinical Electrophysiology. 17 (10), 1603-1609 (1994).

16. Elzinga, W. E. Ameroid constrictor: uniform closure rates and a calibration procedure. Journal of applied physiology. 27 (3), 419-421(1969).

17. Hughes, G. C., Post, M. J., Simons, M., Annex, B. H. Translational physiology: Porcine models of human coronary artery disease: Implications for preclinical trials of therapeutic angiogenesis. Journal of Applied Physiology. 94 (5), 1689-1701 (2003).

18. Lichtig, C., Brooks, H., Chassagne, G., Glagov, S., Wissler, R. W. Basic fuchsin picric acid method to detect acute myocardial ischemia. An experimental study in swine. Archives of Pathology and Laboratory Medicine. 99 (3), 158-161 (1975).

19. Näslund, U., Häggmark, S., Johansson, G., Pennert, K., Reiz, S., Marklund, S. L. Effects of reperfusion and superoxide dismutase on myocardial infarct size in a closed chest pig model. Cardiovascular Research. 26 (2), 170-178 (1992).

20. Reffelmann, T. et al. A novel minimal-invasive model of chronic myocardial infarction in swine. Coronary Artery Disease. 15 (1), 7-12 (2004).

21. Reimer, K. A., Lowe, J. E., Rasmussen, M. M., Jennings, R. B. The wavefront phenomenon of ischemic cell 
death. 1. Myocardial infarct size vs duration of coronary occlusion in dogs. Circulation. 56 (5), 786-794 (1977).

22. Salazar, A. E. Experimental myocardial infarction. Induction of coronary thrombosis in the intact closedchest dog. Circulation research. 9, 1351-1356 (1961).

23. Takahashi, M. et al. Effects of angiotensin I-converting enzyme inhibitor and angiotensin II type 1 receptor blocker on the right ventricular sarcoglycans and dystrophin after left coronary artery ligation. European Journal of Pharmacology. 522 (1-3), 84-93 (2005).

24. Gonzalez-Tendero, A. et al. Whole heart detailed and quantitative anatomy,myofibre structure and vasculature from X-ray phase-contrast synchrotron radiationbasedmicro computed tomography. European Heart Journal Cardiovascular Imaging. 18 (7), 732-741 (2017).

25. Teh, I. et al. Resolving fine cardiac structures in rats with high-resolution diffusion tensor imaging. Scientific Reports. 6, 30573 (2016).

26. Teh, I. et al. Validation of diffusion tensor MRI measurements of cardiac microstructure with structure tensor synchrotron radiation imaging. Journal of Cardiovascular Magnetic Resonance. 19 (1), 31 (2017).

27. Abouezzeddine, $O$. et al. Relevance of endocavitary structures in ablation procedures for ventricular tachycardia. Journal of Cardiovascular Electrophysiology. 21 (3), 245-254 (2010).

28. Pambrun, T. et al. Epicardial course of the septopulmonary bundle: Anatomical considerations and clinical implications for roof line completion. Heart Rhythm. 18 (3), 349-357 (2021).

29. Pallares-Lupon, N. et al. Optimizing large organ scale micro computed tomography imaging in pig and human hearts using a novel air-drying technique. bioRxiv. 2021.07.29.454121 (2021).

30. Martins, R. P. et al. Dominant frequency increase rate predicts transition from paroxysmal to longterm persistent atrial fibrillation. Circulation. 129 (14), 1472-1482 (2014).

31. Puchtler, H., Waldrop, F. S., Valentine, L. S. Fluorescence microscopic distinction between elastin and collagen. Histochemie. 35 (1), 17-30 (1973).

32. Walton, R. D. et al. Compartmentalized Structure of the Moderator Band Provides a Unique Substrate for Macroreentrant Ventricular Tachycardia. Circulation: Arrhythmia and Electrophysiology. 11 (8), e005913 (2018).

33. Di Diego, J. M., Sicouri, S., Myles, R. C., Burton, F. L., Smith, G. L., Antzelevitch, C. Optical and electrical recordings from isolated coronary-perfused ventricular wedge preparations. Journal of Molecular and Cellular Cardiology. 54, 53-64 (2013).

34. Pauwels, E., Van Loo, D., Cornillie, P., Brabant, L., Van Hoorebeke, L. An exploratory study of contrast agents for soft tissue visualization by means of high resolution X-ray computed tomography imaging. Journal of Microscopy. 250 (1), 21-31(2013).

35. Mulet, A. Book Review: Modern Drying Technology, Volume 3: Product Quality and Formulation, edited by E. Tsotsas and A. S. Mujumdar . Drying Technology. 32 (2), 244-245 (2014).

36. Karimi, B., Golshani, B. Mild and highly efficient method for the silylation of alcohols using hexamethyldisilazane catalyzed by iodine under nearly neutral reaction conditions. Journal of Organic Chemistry. 65 (21), 7228-7230 (2000). 
37. Dunmore-Buyze, P. J. et al. Three-dimensional imaging of the mouse heart and vasculature using micro-CT and whole-body perfusion of iodine or phosphotungstic acid. Contrast Media and Molecular Imaging. 9 (5), 383-390 (2014).

38. Disney, C. M., Madi, K., Bodey, A. J., Lee, P. D., Hoyland, J. A., Sherratt, M. J. Visualising the 3D microstructure of stained and native intervertebral discs using $X-$ ray microtomography. Scientific Reports. 7 (1), 16279 (2017).

39. Descamps, E., Sochacka, A., de Kegel, B., Loo, D. Van, Hoorebeke, L., Adriaens, D. Soft tissue discrimination with contrast agents using micro-ct scanning. Belgian Journal of Zoology. 144 (1) (2014).

40. Buytaert, J., Goyens, J., De Greef, D., Aerts, P., Dirckx, J. Volume shrinkage of bone, brain and muscle tissue in sample preparation for micro-CT and light sheet fluorescence microscopy (LSFM). Microscopy and Microanalysis. 20 (4), 1208-1217 (2014).

41. Nierenberger, M., Rémond, Y., Ahzi, S., Choquet, P. Assessing the three-dimensional collagen network in soft tissues using contrast agents and high resolution microCT: Application to porcine iliac veins. Comptes Rendus Biologies. 338 (7), 425-433 (2015).

42. Speck, U. General principles of $x$-ray contrast media.XRay Contrast Media. Springer, Berlin, Heidelberg (2018).

43. Rajasekar, A., Trew, M. L., Sands, G. B. Understanding and enhancing the use of micro-computed tomography in soft tissue. University of Auckland, Auckland (2015).

44. Karagiannidis, E. et al. Micro-CT-based quantification of extracted thrombus burden characteristics and association with angiographic outcomes in patients with
ST-elevation myocardial infarction: The QUEST-STEMI Study. Frontiers in Cardiovascular Medicine. 8, 646064 (2021).

45. Karagiannidis, E. et al. Serum ceramides as prognostic biomarkers of large thrombus burden in patients with stemi: A micro-computed tomography study. Journal of Personalized Medicine. 11 (2), 89 (2021).

46. Hennig, A. et al. High-resolution three-dimensional late gadolinium-enhanced cardiac magnetic resonance imaging to identify the underlying substrate of ventricular arrhythmia. Europace: European Pacing, Arrhythmias, and Cardiac Electrophysiology: Journal of the Working Groups on Cardiac Pacing, Arrhythmias, and Cardiac Cellular Electrophysiology of the European Society of Cardiology. 20 (FI2), f179-f191 (2018).

47. Lorgis, L. et al. Relationship between fragmented QRS and no-reflow, infarct size, and peri-infarct zone assessed using cardiac magnetic resonance in patients with myocardial infarction. Canadian Journal of Cardiology. 30 (2), 204-210 (2014). 